\title{
PRETERM BIRTH (PTB) AND CONGENITAL HEART DEFECTS (CHD): A POPULATION- BASED STUDY
}

\author{
E. Laas ${ }^{1}$, N. Lelong ${ }^{1}$, A.-C. Thieulin ${ }^{1}$, L. Houyel ${ }^{2}$, D. Bonnet ${ }^{3}$, P.-Y. Ancel ${ }^{1}$, G. Kayem ${ }^{1}$, F. Goffinet ${ }^{1,4}$, B. \\ Khoshnood $^{1}$, EPICARD \\ ${ }^{I}$ INSERM U953, Paris, ${ }^{2}$ Service de Chirurgie des Cardiopathies Congénitales, Hopital Marie Lannelongue, \\ Le Plessis Robinson, ${ }^{3}$ Cenre de Référence M3C-Necker, Université Paris Descartes, ${ }^{4}$ Maternité Port-Royal, \\ Hopital Cochin-Saint Vincent de Paul, Paris, France
}

Background and aims: PTB and CHD are the two main causes of mortality, morbidity and disability of perinatal origin. There are little population-based data on the relation between CHD and PTB.The objective of this study was to estimate the prevalence of prematurity in newborns with CHD and to study specific associations between subcategories of CHD and PTB.

Methods: We used data from the EPICARD study, a population-based cohort study of CHD in France, including 2349 livebirths with CHD. We used an anatomic and clinical classification of CHD based on the International Paediatric and Congenital Cardiac Code to categorise CHD into ten groups. Data on PTB from the French National Perinatal Survey were used to compare PTB in EPICARD to that of the general population.

Results: $15 \%$ (95\%CI, 13.6-16.5) of the newborns with CHD were PTB and the odds of PTB was more than two fold higher than for general population (OR $2.395 \% \mathrm{CI}, 1.8-2.8$ ). The higher risk of PTB, and particulary very PTB ( $<32$ weeks), associated with CHD persisted after exclusion of cases associated with chromosomal anomalies and/or non-cardiac defects and adjustement for maternal age, sociodemographic characteristics and diabetes. There were wide variations in PTB across the ten groups examined.

Conclusion: In this population-based study, the risk of PTB and particulary very PTB was substancially higher for newborn with CHD. Risk of PTB varied highly for subcategories of CHD and the specific associations oberved may be helpful for generating hypotheses about the developemental links between CHD and PTB. 